\title{
How to write a scientific paper? Lessons from a distinguished scientist and editor
}

\author{
Shan Ali $^{{ }^{*}}$ (1) , Tomasz Szmuda ${ }^{2 *}$, Z Zbigniew K. Wszołek ${ }^{3 *}$ (i)
}

${ }^{1}$ Scientific Circle of Neurology and Neurosurgery, Neurosurgery Department, Medical University of Gdańsk, Poland ${ }^{2}$ Department of Neurosurgery, Medical University of Gdańsk, Gdańsk, Poland

${ }^{3}$ Department of Neurology, Mayo Clinic in Florida, Jacksonville, FL, USA

*all authors contributed equally to the manuscript

\begin{abstract}
This article is an instructive guide on how to write and publish a scientific article. It was inspired by a lecture given at the Medical University of Gdańsk by a distinguished professor. To further advance science, particularly in the currently emerging era of individualized medicine, the collaboration of researchers with a varied level of experience and from different areas of expertise is needed. It is vital to publish the results of research quickly and effectively. Knowing the basic mechanisms of writing and publishing will help to disseminate research results more effectively.
\end{abstract}

Keywords: neuroscience $\cdot$ publishing $\cdot$ scientists $\cdot$ neurology $\cdot$ neurosurgery $\cdot$ generations

\section{Citation}

Ali S., Szmuda T., Wszołek ZK. How to write a scientific paper? Lessons from a distinguished scientist and editor. Eur J Transl Clin Med. 2020;3(1):74-78.

DOI: $10.31373 /$ ejtcm/118954

This article is an instructive guide on scientific writing and successful publication. It was inspired by professor Zbigniew Wszołek's keynote lecture (presented on May 28, 2019 at the Medical University of Gdańsk) on the subject How to write and publish a scientific paper?

\section{How to start writing?}

When writing the manuscript, one may choose to write in the following order: results, methods and materials, introduction, discussion, conclusion and the abstract. In this way, the author is 'forced' to analyze

Shan Ali, Scientific Circle of Neurology and Neurosurgery, Neurosurgery Department, Medical University of Gdańsk, Poland

e-mail: shanali@gumed.edu.pl

No external funds.

Available online: www.ejtcm.gumed.edu.pl

Copyright ${ }^{\circledR}$ Medical University of Gdańsk

This is Open Access article distributed under the terms of the Creative Commons Attribution-ShareAlike 4.0 International. 
all the data for any significant findings and to formulate the main thesis of the paper before doing most of the writing. However, the final version needs to follow the universally-accepted structure: first the abstract, then the introduction, materials and methods, results, discussion, and last but not least the conclusion.

\section{Authorship}

If two authors contributed equally to a publication, they may feel free to share first authorship [1]. In 2012 almost $30 \%$ of all medical publications had co-first authorship [2]. For instance, this editorial shares first-authorship between the three authors. Placing the most senior investigator as the last author is customary, however according to the current guidelines if the senior investigator's role consisted of project supervision only, then s/he should be just mentioned in the acknowledgement section [3]. Group authorship (Group Corporate Authorship) instead of single authors is a possibility and is recognized by the National Library of Medicine database [4].

\section{The Abstract}

The abstract is a crucial part of a manuscript because it provides the initial impression for the reader. Scientific journals require that the abstract is limited to about 200 to 300 words and is a separate part of the manuscript. The most significant and most important parts of the paper are usually presented in the abstract. It is typically arranged in the following sections: background, aim/purpose, material and methods, results and conclusion.

\section{The Introduction}

The introduction is a short and concise review of your topic and is directly relevant to the aim of your study. It follows a standard format and should not be longer than three paragraphs. The first paragraph focuses on information that is already known and established. The second paragraph introduces the knowledge gap or the limitations of current scientific knowledge. The third paragraph declares the purpose of the study and why the study may be clinically relevant. Notably, the introduction should not yet answer the main research question and should not contain any results.

\section{Ethical Commitee Approval \& Patient Consent}

Ethical committee approval and patient consent is required for almost any study involving people or their information (e.g. responses on questionnaires). Obtaining approval may take several months so we recommend starting this process early. Only data that is publicly available (such as online information from the World Health Organization) does not require official approval for use in research. If author/s have any specific questions or concerns particularly related to the obtaining permissions for publication, it is advisable they seek the guidance of the local Ethics Committee.

\section{The Material and Methods}

This section is a detailed description of how the material was obtained and what test or experiments were performed. There should be enough details mentioned to make it possible to replicate the described study. It is often helpful to divide this section with headings (e.g. inclusion criteria, exclusion criteria, experimental protocol and statistical methods). Instead of writing detailed descriptions of commonly used methods or protocols, authors may simply cite a previous study that involved the same methods. Flowchart diagrams may be useful for describing complex study designs.

\section{The Results}

This section summarizes the data with significant relationships and the overall trends and may be supported by tables or graphs. Like the material and methods section, when necessary the results section may be divided into subsections with headings. Only the most important data should be repeated from the tables and should be analyzed when possible (e.g. the percentage change instead of absolute values). In this section, the term "significant" should be only used to mean "statistically significant." Analysis of your data should be reserved for the discussion section. All data should be presented, including non-significant findings and negative results. We recommend making one's findings as transparent as possible by providing supplementary raw data as it may encourage confidence in a reader [5]. Avoid making the common mistake which is mixing the results with the methods and materials.

\section{The Discussion}

The discussion consists of six basic parts: the key findings, context, limitations, future outlook, clinical implications and the conclusion.

1. The key findings state what the data means and if the findings are novel. This information should be summarized to the extent that even a non-expert 
may understand the relevance of the findings to to health and/or science.

2. In the context section, the authors compare their results with the most recent scientific literature. This section should explicitly state whether your findings confirm or challenge the current paradigm. Here, you may discuss the results or offer possible mechanisms for your findings.

3. The limitations section is obligatory and is a chance for the author(s) to respond to the criticism that they anticipate from the peer-reviewers and of course the readers. This section should convince the reader that despite their limitations, your results are relevant.

4. In the future outlook section, the authors may recommend confirmatory studies and suggest the direction/s of follow-up studies.

5. The clinical implications section outlines the impact that the research results may have on clinical practice. In other words, answer the question "why should physicians pay attention to the findings of the paper?"

6. The conclusion is where the main findings are emphasized and the final message is restated.

\section{Citations}

Every publisher aims to increase the impact factor of its journals (which is measured by the number of citations a journal obtains over the last few years) [6]. Thus, it is in the interest of the author to cite papers published in the journal they are sending their manuscript to.

\section{Plagiarism}

We encourage authors to check for plagiarism before submitting a manuscript. Quoting other papers is fine, however, to help you and your co-authors distinguish it from your original text we recommend placing it in bold or italics. Auto-plagiarism, a situation when an author copies his/her fragment from a previously published article, is also prohibited.

\section{Language}

We recommend that a researcher not pay excessive attention to the language, style and grammar for the first draft. In the first draft the goal is to write the main, technical/scientific content of the paper, whereas the grammatical and stylistic improvements should be left for later revisions. To improve the writing, we suggest regularly reading scientific journals relevant to one's own research interest in order to become familiar with the correct and accepted vocabulary, phrasing and sentence structure.
We recommend authors whose native language is not English to avoid direct translations from their native language to English because this often results in confusing or awkward phrases and incorrect sentence structure.

Revise the manuscript several times before final submission, with the intention of making technical topics easy to understand and pleasant to read even for non-experts. The manuscript's final version should contain convincing arguments with no grammatical mistakes. When expressing opinion/s, authors should remain professional, polite, fair and avoid emotionally-charged words and phrases.

\section{Choosing the Journal}

When choosing the journal to submit your manuscript, keep in mind not only its impact factor but its scope (e.g. a paper on stroke treatment should not be sent to a movement disorders journal). We recommend browsing the latest issue of a journal to gain an understanding of what types of articles are actually published there.

\section{The Submission Process}

We encourage authors to identify their target journal before they start writing their paper so that they are aware of the author guidelines. These provide information about the word limit, number and format of the tables or figures, style of references, conflict of interest forms, et cetera. A manuscript may be rejected at the initial submission stage if it does not meet the journal's requirements, therefore adhering to these guidelines is one of the keys to successful publishing. After you submit your manuscript on the journal's online system the following outcomes are possible: accepted; accepted pending/with minor revisions; accepted pending/with major revisions; rejected but re-submission possible; rejected with no resubmission possible. Most papers will require some revisions, more about that later.

\section{The Cover Letter}

The cover letter is often an unappreciated part of one's submission as it offers the opportunity to tell the Editor something that cannot be stated in the manuscript. If it is well-written, it may help convince the Editors to publish the manuscript. However, a well-written cover letter will not 'save' a poorly-written manuscript.

\section{Writing the Rebuttal Letter}

The rebuttal letter explains exactly what the authors changed to improve the paper according to the reviewers' comments. Occasionally, reviewers may not fully understand the context of your manuscript. 
Regardless, it is important to write your rebuttal in a polite tone. Always state that you appreciate the insightful comments of the reviewers and that their input helped you improve your manuscript and now it is more relevant in your field of study.

\section{Publishing Case Reports}

Case reports are relatively easy and quick to write; however, they are notoriously difficult to publish. The reason for this is simple: most medical journals do not publish case reports. Therefore, in order to be accepted for publishing, the case report must be truly exceptional and novel. We encourage scientists to first find a respected journal that publishes case reports on a given topic. We warn scientists to avoid the numerous predatory journals which advertise that they publish case reports just to make a profit (by charging the authors article processing fees). Such journals can be identified by referencing one of the publicly available online lists of predatory journals.

\section{Conducting Research as a Student}

As a student without a laboratory, research funding or any patients of one's own, it might seem rather impossible to conduct independent research. Fortunately, there are several options:

1. Systematic reviews (with or without a meta-analysis) are an excellent way of gaining fairly high-impact publications without having one's own laboratory [7]. In fact, the most referenced studies on a given topic are usually provided by systematic reviews and not original papers [8]. Through the writing process, one may learn how to gather, manage and interpret other scientist's findings. The process allows a scientist to conduct an in-depth analysis of previous studies on a particular topic which may help them find something of significance that has not been yet researched.

2. Papers on infodemiology (the epidemiology of information) may be done without a laboratory. One may analyze online health patterns for a particular disease and then relate it to the epidemiology of the disease in a population. Studies such as this may illuminate the overall health seeking behavior in a population [9-11]. Freely accessible databases provided by the World Health Organization, the Centre for Disease Control and Prevention and a country's own health registries are invaluable in these types of papers. Furthermore, with the era of the internet, students can conduct content, readability and quality analyses of popular web platforms (i.e. YouTube, Wikipedia, and Google search results) for a particular disease or topic so that scientists are aware of the quality of information that the public is reading.

3. Letters to the Editor may be written on a recently published article (usually within one month). These letters serve or to augment the understanding of an article or to simply criticize the study. For example, one may write a letter pointing out the significant limitations of a study that were not pointed out in the actual article [12]. One may also analyze the parts of the paper differently [13-15].

4. Several highly respected journals (e.g. JAMA, JAMA Oncology, Annals of Internal Medicine and Journal of Clinical Oncology) feature a humanities column where poetry, personal vignettes and essays may be published on the medical experience [16-18]. These articles often comment on the dynamics of the patient-physician relationship and may offer a social critique of the medical system.

\section{Conclusion}

Writing and publishing a scientific article is an ongoing process requiring several revisions and often months of dedication. Although the process can be challenging, we believe it is an incredibly gratifying experience especially with the help of co-authors who share the same passion for medicine. We hope that this guide will be of use to students, residents, fellows, and young faculty members.

1. Lapidow A, Scudder P. Shared first authorship [Internet]. Vol. 107, Journal of the Medical Library Association. Medical Library Association; 2019 [cited 2020 Mar 3]. p. 618-20. Available from: http://www.ncbi.nlm.nih.gov/pubmed/31607826

2. Conte ML, Maat SL, Bishr Omary M. Increased co-first authorships in biomedical and clinical publications: A call for recognition [Internet]. Vol. 27, FASEB Journal. FASEB; 2013 [cited 2020 Mar 3]. p. 3902-4. Available from: http://www.ncbi. 
3. Baerlocher MO, Newton M, Gautam T, Tomlinson G, Detsky AS. The meaning of author order in medical research. J Investig Med [Internet]. 2007 May [cited 2020 Mar 3];55(4):174-80. Available from: http://www.ncbi.nlm.nih.gov/pubmed/17651671

4. Authorship in MEDLINE [Internet]. U.S. National Library of Medicine; [cited 2020 Mar 3]. Available from: https://www. nlm.nih.gov/bsd/policy/authorship.html

5. Chan AW, Song F, Vickers A, Jefferson T, Dickersin K, Gøtzsche PC, et al. Increasing value and reducing waste: Addressing inaccessible research [Internet]. Vol. 383, The Lancet. Lancet Publishing Group; 2014 [cited 2020 Mar 3]. p. 257-66. Available from: http://www.ncbi.nIm.nih.gov/pubmed/24411650

6. Wszolek ZK, Sławek J, Siemiński M. Current Status of the Polish Journal of Neurology \& Neurosurgery (Neurologia i Neurochirurgia Polska). Neurol Neurochir Pol [Internet]. 2019;53(3). Available from: https://journals.viamedica.pl/neurologia neurochirurgia polska/article/download/64902/48655

7. Szmuda T, Sloniewski P, Waszak PM, Springer J, Szmuda M. Towards a new treatment paradigm for ruptured blood blister-like aneurysms of the internal carotid artery? A rapid systematic review [Internet]. Vol. 8, Journal of Neurolnterventional Surgery. BMJ Publishing Group; 2016 [cited 2020 Mar 3]. p. 488-94. Available from: http://www.ncbi.nlm.nih.gov/ pubmed/25792038

8. Yeung AWK, Goto TK, Leung WK. At the leading front of neuroscience: A bibliometric study of the 100 most-cited articles. Front Hum Neurosci [Internet]. 2017 Jul 21 [cited 2020 Mar 3];11:363. Available from: http://www.ncbi.nlm.nih.gov/ pubmed/28785211

9. Szmuda T, Ali S, Stoniewski P. How useful is Google Trends in evaluating public interest in neurosurgical diseases? (Preprint). JMIR Prepr [Internet]. 2019 Oct 20; Available from: https://doi.org/10.2196/preprints.16743

10. Szmuda T, Ali S, Słoniewski P. Are Wikipedia statistics useful in evaluating public interest in neurosurgical diseases? (Preprint). JMIR Prepr [Internet]. 2019 Oct 20; Available from: https://doi.org/10.2196/preprints.16742

11. Szmuda T, Ali S, Słoniewski P. Assessing neurosurgical trends in literature and online: a Google Trends, Google Books and PubMed study (Preprint). JMIR Prepr [Internet]. 2019 Oct 25; Available from: https://doi.org/10.2196/preprints.16798

12. Szmuda T, Ali S, Stoniewski P. Letter to the Editor Regarding "A Quality Analysis of Disk Herniation Videos on YouTube." World Neurosurg. 2019;130.

13. Szmuda T, Ali S, Słoniewski P. Letter to the Editor. Harvey Cushing's legacy. J Neurosurg [Internet]. 2019 Sep; Available from: https://www.ncbi.nlm.nih.gov/pubmed/31561216

14. Szmuda T, Ali S, Słoniewski P. Letter to the Editor. Dr. Dwight Parkinson's legacy. J Neurosurg [Internet]. 2020 Jan 10 [cited 2020 Mar 3];1-2. Available from: http://www.ncbi.nlm.nih.gov/pubmed/31923889

15. Szmuda T, Ali S. Commentary on: The usefulness and limitations of diffusion tensor imaging - a review study. Eur J Transl Clin Med. 2019;0.

16. Ali S. Medicine Is Not a Subway Ride. JAMA Oncol [Internet]. 2020 Feb 27 [cited 2020 Mar 3]; Available from: https:// www.ncbi.nlm.nih.gov/pubmed/32105295

17. Ali S. Delivering Good News. JAMA Oncol [Internet]. 2020 Feb 1 [cited 2020 Mar 3];6(2):303. Available from: https:// www.ncbi.nlm.nih.gov/pubmed/31876944

18. Ali S. A Resident's Courage. JAMA Oncol [Internet]. 2020 Jan 1 [cited 2020 Mar 3];6(1):163. Available from: http://www. ncbi.nlm.nih.gov/pubmed/31697375 